\title{
Optimal placement of distribution transformers in radial distribution system
}

\author{
Vishwanath Hegde*, Raghavendra C. G., Prashanth Nayak \\ Pradeep S., Themchan Woleng \\ Department of Electrical and Electronics Engineering, Malnad College of Engineering, Hassan -573201, Karnataka, India
}

\begin{abstract}
The global electricity industry is facing many challenges that require immediate attention towards growth, expansion and energy management Most of the electricity supply companies are facing shortfall of availability of electrical energy. Therefore to make up for the shortfall of the electrical energy availability, reducing losses and hence saving energy becomes the first and foremost important step as a part of demand side management. Application of smart grid technologies is one of the key tools in solving the problems faced by electricity industry. In this direction determination of optimal location of the distribution transformer is an important issue to be addressed. The electricity supply companies do not seem to use any proved method to find the optimal location of the distribution transformer. Moreover in many cases distribution transformers can not be placed in the optimal position due to socio-political and economical considerations which will result in higher losses.

In the present work an attempt has been made to find the optimal location of a distribution transformer based upon the minimum peak power loss and minimum voltage regulation using MiPower software and the result obtained has been compared with the node elimination method and the results are found to be encouraging with respect to theoretically calculated values.
\end{abstract}

Keywords: Distribution transformer, voltage regulation, MiPower, node elimination method

\section{Introduction}

The electrical energy is an important factor in the economical and social growth of any country in maintaining and developing a modern economy and society. Economical, social and environmental sustainability are the most important variables in the energy scenario of the 21 st century [1]. Utilities are facing many difficult challenges because of environmental awareness, rigorous regulations, growing demand for high-quality supply and rising customer expectations [2], [3]. From the bottom up, the restructure of electricity generation, transmission and distribution system is required for the efficient usage and management of available energy. In the world, the smart grid (SG) technologies are becoming highly important to solve the energy crisis. The smart grid is now-a-days often called intelligent grid, which has the power delivery integration and the high level of utility strategic planning functions. At present, energy conservation sustainable development, safety, reduction of loss, optimal utilization of assets become the main attention. Considering the modern technological innovations and trends, the implementation of the smart grid is possible in the world's electric power system to obtain a flexible, clean, safe, economical, and friendly intelligent grid.

The optimum distribution system planning is one of the most important and critical tasks in power system planning. This problem is so complex that the task is divided into sub tasks as distribution transformer allocation and feeder allocation. In distribution planning, first the peak load magnitude and its geographic location must be determined, then the optimal location and size of distribution transformer

\footnotetext{
* Manuscript received May 30, 2013; revised July 28, 2013.

Corresponding author. Tel.: +91-9449207412; E-mail address: hedge_mce@rediffmail.com.
} 
must be determined in such a way as to supply all load points at minimum loss, voltage regulation and cost. This not only serves for finding out the losses in the system and improving the system performance, but also helps in maintaining the quality of services to the customer. In India, the total transmission losses are found to be $10 \%$ and the distribution losses are found to be in the range of $20 \%$ to $30 \%$ [4]. This is too high when compared to the combined losses in developed countries like U.S.A Canada, Singapore etc. Thus there is a need to reduce the losses especially in the distribution system where losses are too high, by adopting some economical and efficient techniques.

Marlin and Bak [5] have first proposed a feeder reconfiguration technique for loss minimization in distribution system. In this method all the network switches are closed to form a closed system, and then the switches are opened successively, to restore to the radial configuration, thus minimizing the losses. However, this method involves approximations. Peponis et al. [6] have proposed a technique for the optimal operation of distribution network. In this method shunt capacitors are installed for loss minimization and reconfiguration of the network. Schmidt et al. [7] have formulated the problem as a mixed integer nonlinear optimization problem. Morton et al. [8] have proposed a method based on an exhaustive search algorithm for obtaining a minimum loss of a radial distribution system. M.W. Siti et al. [9] have proposed a technique at the low-voltage and medium-voltage levels of a distribution network with reconfiguration at both levels. K. Viswanadha Raju et al. [10] have proposed a new, two stage, and heuristic method for determining a minimum loss configuration of a distribution network, based on real power loss sensitivities with respect to the impedances of the candidate branches. Javad Olamaei et al. [11] have proposed distribution feeder reconfiguration for loss minimization using modified honey bee mating optimization algorithm. Ali Arefi et al. [12] have discussed the experiences of loss reduction projects in electrical power distribution companies of Iran as a function of cost reduction process.

From the above literature it can be concluded that distribution losses account for a major portion of the technical losses. Most of the methods available for this purpose may not be economical and they may not give an immediate solution. So there is a need to adopt an economical method like relocation/optimal placement of distribution transformer which gives an immediate solution.

Before a new Distribution Transformer Centre (DTC) is to be installed or the installed DTC has to be relocated, it is very much necessary to find the optimal location (feed point) of the DTC corresponding to minimum losses and minimum voltage regulation. Hence the objective of the present work is to find the optimal location of the DTC (TC Code: 413A11) in a practical 9 bus, $415 \mathrm{~V}$, radial distribution system using MiPower [13] software package and to compare the results obtained with that of node elimination method. Thus the present work verifies the existing location of the DTC (TC Code: 413A11, $250 \mathrm{kVA}, 11$ $\mathrm{kV} / 415$ volts, $50 \mathrm{~Hz}$ ) located at Bangalore - Mangalore Road, Hassan, for optimal location.

\section{Methodology}

It is generally known that the feed point located near the "load center" minimizes the losses. The methods presently in use for locating such load centers are generally empirical and based on either 'Rule of Thumb' or heuristic methods which are not efficient methods. Here, an alternate method for determining the feed point has been employed. This method requires effectively only one network solution irrespective of the system size and computationally more efficient as compared to other methods. In addition, it is shown that the solution obtained is indeed the optimal solution [14]. The algorithm used here is essentially a systematic search procedure which considers all the nodes of the network as possible choices for the feed-point, eliminates one node at a time from the candidate list and ultimately locates the optimal location at the end of $\mathrm{N}$ steps. In formulating the problem of optimal location of the DTC, it is assumed that the locations and values of all the loads are known, the network configuration remains unaltered and the total load of the network is less than the capacity of a single transformer.

\subsection{Algorithm to find the optimal location}

This algorithm is intended to locate the optimal feed point among all the network nodes so as to minimize the peak power losses in the system. The algorithm used here considers all the nodes of the 
network, one by one, and locates the optimum in $\mathrm{N}$ steps. The algorithm is developed by exploiting some of the special features of the distribution networks. In particular, the absence of loops and the current through any segment is uniquely determined depending on the direction of the current flow through the segment irrespective of the position of the feed point [14].

Algorithm

1. Compute the load currents at all nodes. Determine the set of terminal nodes. Set $i=N$.

2. Determine the terminal node, T, having the minimum load current, $I_{\min }$.

3. Increment the load current of the source node $S$, to which $T$ is directly connected, by a value equal to $I_{\text {min }}$.

4. Eliminate node $\mathrm{T}$ and the edge connecting $\mathrm{S}$ and $\mathrm{T}$.

5. Update the list of terminal nodes. Set $i=i-1$.

6. If $i \geq 1$ go to step 2 .

7. Output the remaining node as the optimal feed point.

\subsection{Simulation using MiPower package}

MiPower is a highly interactive, user friendly window based Power System Analysis package. It includes a set of programs for performing a range of power system design and analysis study. MiPower features include a top notch Windows GUI with network editor and single line diagram generation. It includes the application programs like Load Flow Analysis, Short Circuit, transient Stability, Relay Coordination, Harmonic Analysis, Long Term Load Forecast etc [13].

\subsection{Optimal location corresponding to minimum losses}

The single line diagram of the distribution network will be generated using MiPower package and the database for the same will be configured. Generator and Transformer of rating slightly greater than total connected load will be considered for the purpose of simulation. Then each bus of the network is considered as a possible feed point by connecting the distribution transformer to every bus one after the other and the load flow studies using Newton Raphson method are made to obtain the bus voltages and total losses of the system. Thus the optimal location of DTC corresponding to minimum losses is obtained in MiPower package.

\subsection{Optimal location corresponding to minimum voltage regulation}

Optimal location corresponding to minimum losses (say feed point A) obtained may not be 'Optimal' from the point of view of the voltage regulation. In order to find the optimal location corresponding to minimum voltage regulation and thus the final optimal position of the considered DTC, voltage drops need to be calculated to all tail ends from A. If, at all the tail ends the voltage criterion (i.e. maximum voltage regulation of $6 \%$ [15]) is satisfied, then $\mathrm{A}$ is the optimal feed point. Otherwise the feed point corresponding to next minimum losses has to be verified.

\subsection{Optimal placement of distribution transformer}

It is important to have an accurate look at the problem of allocation of DTC. The installation of DTC at non-optimal places can result in an increase in system losses, increase in costs and therefore having an effect opposite to the desired. Therefore, the use of methodology capable of analyzing the influence of system characteristics of DTC allocation can be very effective for system planning engineer.

Here, the optimal location for placing the DTC is determined by looking into the losses and voltage regulation at various feed points.

\section{Case Study and Simulation Results}

To analyze the optimal location of a distribution transformer, a $415 \mathrm{~V}, 9$ nodes radial distribution 
system whose feed point is at bus 1 is considered and as shown in Fig. 1. For this system, the loads (kW, $\mathrm{kVAr}$ ), as well as the currents at all the nodes, are given in Table 1, and lengths of all the segments are taken equal to 40 meter. The resistance and reactance of the conductor used are $1.578 \mathrm{Ohm}$ and 0.354 Ohm per km, respectively [16]. The maximum allowable voltage drop is 0.06 p.u. The one line diagram of the system considered is shown in Fig. 1, wherein the nodes are labelled by numbers.

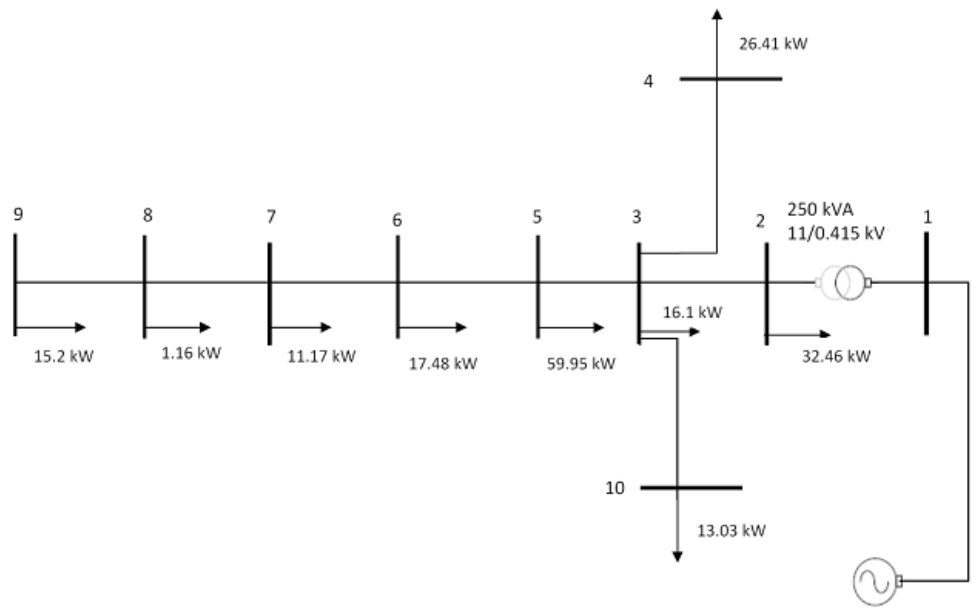

Fig. 1. Single line diagram of the 9 bus, $415 \mathrm{~V}$ radial distribution system.

Table 1. Load data of the 9-node radial system

\begin{tabular}{clll}
\hline \hline \multirow{2}{*}{ Node number } & \multicolumn{2}{c}{ Load } & Current \\
& $P(\mathrm{~kW})$ & $Q(\mathrm{kVar})$ & $(\mathrm{A})$ \\
\hline 2 & 32.46 & 20.17 & 45.15 \\
3 & 16.1 & 9.97 & 22.39 \\
4 & 26.41 & 16.37 & 36.74 \\
5 & 59.95 & 37.15 & 83.40 \\
6 & 17.48 & 10.83 & 24.31 \\
7 & 11.17 & 6.92 & 15.54 \\
8 & 1.16 & 0.7189 & 1.61 \\
9 & 15.2 & 9.42 & 21.14 \\
10 & 13.03 & 8.07 & 18.13 \\
\hline \hline
\end{tabular}

Table 2. 9 Node systems: node elimination sequences

\begin{tabular}{lccc}
\hline \hline Step number & Terminal nodes & Terminal node currents (A) & Node eliminated \\
\hline 1 & $9,4,10,2$ & $21.14,36.74,18.13,45.15$ & 10 \\
2 & $9,4,2$ & $21.14,36.74,45.15$ & 9 \\
3 & $8,4,2$ & $22.75,36.74,45.15$ & 8 \\
4 & $7,4,2$ & $38.29,36.74,45.15$ & 4 \\
5 & 7,2 & $38.29,45.15$ & 7 \\
6 & 6,2 & $62.6,45.15$ & 2 \\
7 & 6,3 & $62.6,67.54$ & 6 \\
8 & 5,3 & $146,67.54$ & 3 \\
9 & 5 & ------ & - \\
\hline \hline
\end{tabular}

\subsection{Node Elimination method}

The Optimal location of the DTC can be obtained theoretically using node elimination sequence.

The details of typical steps of the search procedure (Algorithm) considering the first step are given below.

i) The set of terminal nodes of the system is $\{2,4,9,10\}$

ii) The terminal node having minimum current is found using Table 2 as 10 .

iii) Increment the node current of node, the source node, corresponding to the terminal node by $I_{1}$ (amps). 
iv) Eliminate node and the corresponding segment connecting nodes and

v) The updated set of terminal nodes would be $\{2,4,9\}$

The above sequence of steps is repeated 9 times. The critical parameters at each step of the above process are summarized in Table 2. In the last step, it is seen that the single terminal node left is 5 and from the point of view of minimum losses this is the optimal feed point.

\subsection{Simulation using MiPower}

The 9 bus system was simulated in MiPower package and the results obtained are discussed as below.

\subsection{Optimal location (feed point) corresponding to minimum losses}

The simplified network of $415 \mathrm{~V}, 9$ bus Radial distribution system with DTC ( $250 \mathrm{kVA}$ ) is generated using MiPower package and the database for the same is configured. Generator and Transformer of rating $250 \mathrm{kVA}$ were considered for the purpose of simulation. Then each bus of the network is considered as a possible feed point by connecting the DTC to every bus one after the other and the load flow studies were made to obtain the bus voltages and total losses of the system [17]. Thus the optimal location of DTC corresponding to minimum losses was obtained at bus 5 when simulated in MiPower package. Simulated networks with the DTC connected at bus 5 along with the details are shown in Fig. 2.

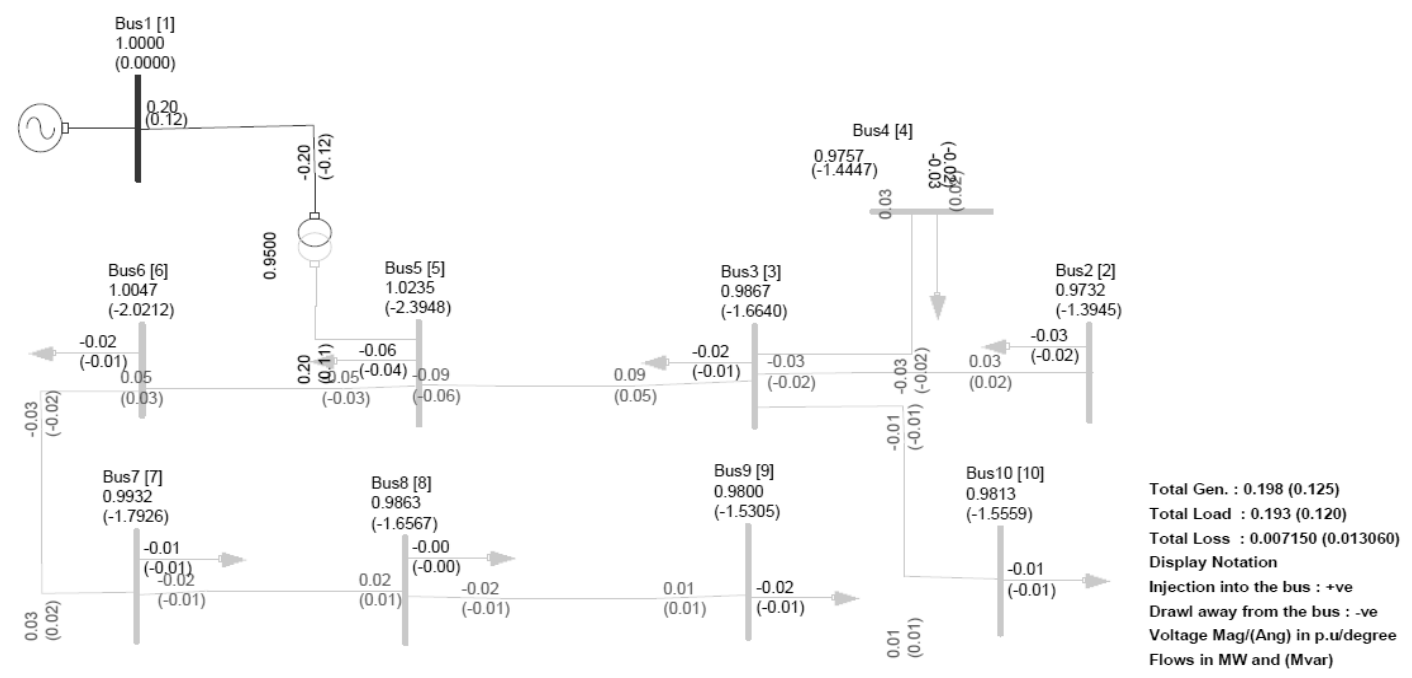

Fig. 2. Simulated network with DTC connected at node 5.

\subsection{Optimal location (feed point) corresponding to minimum voltage regulation}

Optimal location corresponding to minimum losses (node 5) has been obtained but it may not be 'Optimal' from the point of view of the voltage regulation. In order to find the optimal location corresponding to minimum voltage regulation and thus the final optimal position of the considered DTC, voltage drops need to be found to all tail ends from node 5. If, at all the tail ends the voltage criterion (i.e. maximum voltage regulation of $6 \%$ ) is satisfied, then node 5 is the optimal feed point. Otherwise the feed point corresponding to next minimum losses has to be verified. For the 9 bus system considered optimal location corresponding to minimum voltage regulation is found at bus 5 . The minimum loss and minimum voltage regulation obtained using simulation is found to be same as that found in the node elimination method.

\subsection{Optimal placement of distribution transformer}

Since bus 5 corresponds to both minimum losses and minimum voltage regulation, it is considered as the optimal feed point for relocating the DTC from bus 2 . 


\section{Results and Discussion}

Using algorithm, it is found that the optimal location of DTC for 9 bus system is at bus 5 and when the network is simulated using MiPower package, the location corresponding to minimum losses and minimum voltage regulation is obtained when the DTC is relocated to the same location i.e., bus 5. Thus the location of the DTC obtained theoretically matches with that of the simulated result. Losses and voltage regulation obtained in MiPower when the DTC was connected at different buses are as shown in Table 3 and Table 4 respectively. From the Table 4, it can be observed that for 9 bus system when the DTC is connected at bus 5 voltage criterion (maximum voltage regulation of $6 \%$ ) is satisfied at all the terminal buses.

Table 3. Losses at different buses for 9 bus system

\begin{tabular}{cl}
\hline \hline DTC connected to bus & Losses $(\mathrm{kW})$ \\
\hline 2 & 21.93 \\
3 & 8.8 \\
4 & 22.36 \\
5 & 7.15 \\
6 & 17.14 \\
7 & 30.38 \\
8 & 45.58 \\
9 & 60.81 \\
10 & 24.95 \\
\hline \hline
\end{tabular}

Table 4. Percentage Voltage regulation at the terminal buses ( 9 bus system)

\begin{tabular}{ccccc}
\hline \hline $\begin{array}{c}\text { DTC connected } \\
\text { to bus }\end{array}$ & 2 & 4 & 9 & 10 \\
\hline 2 & 0.0 & 7.63 & 15.08 & 7.04 \\
3 & 1.33 & 1.08 & 8.53 & 0.54 \\
4 & 8.12 & 0.00 & 15.32 & 7.33 \\
5 & 4.91 & 4.67 & 4.25 & 4.12 \\
6 & 10.95 & 10.71 & 2.41 & 10.16 \\
7 & 17.69 & 17.45 & 1.29 & 16.90 \\
8 & 24.90 & -1.56 & 0.63 & 24.11 \\
9 & 32.15 & -3.01 & 0.00 & 31.36 \\
10 & 8.67 & 8.42 & 15.87 & 0.00 \\
\hline \hline
\end{tabular}

Therefore from the above discussions, it is concluded that the optimal location of the transformer corresponding to both minimum losses and voltage regulation is at bus 5 .

In the 9 bus Radial distribution system considered, if the DTC is relocated from bus 2 to bus 5 then line losses can be reduced to a greater extent. In case if the DTC cannot be placed at bus 5 due to some socio-political and economical reasons or due to the factors such as topography, land ownership, environmental considerations etc. then the other best possible solution is at bus 3 where the loss is $8.8 \mathrm{~kW}$ (second minimum loss) and voltage criterion is satisfied at all the terminal buses except at buses 9 .

In case if there are more than one distribution transformers to be placed, then based on the load, position of each distribution transformer is to be optimized.

\section{Conclusions}

The present work mainly deals with verifying the optimal location for the existing location of the TC 415A11 located at BM road, Hassan. The location of the TC 415A11 in 9 node Radial distribution system corresponding to minimum loss was obtained theoretically using node elimination sequence, then the location corresponding to minimum loss was obtained by simulation using MiPower package. Then considering this location, the location corresponding to minimum voltage regulation was determined and the location of the DTC corresponding to both minimum loss and voltage regulation is considered as the optimal feed point to relocate the DTC from its original position. 


\section{References}

[1] Acharjee P. Strategy and implementation of smart grids in India. Energy Strategy Reviews, 2012: 1(3):193-204.

[2] Amin SM, Wollenberg BF. Towards a smart grid: power delivery for the 21st century. IEEE Power Energy Mag. 2005; 3(5): 34-41.

[3] Garrity TF. Getting smart. IEEE Power Energy Mag. 2008; 6(2):38-45.

[4] Dinamani K, et al. Measurement of aggregate technical and commercial losses and improvement of distribution system efficiency. B.Tech Dissertation, Malnad College of Engineering, Hassan, 2009.

[5] Merlin, Back H. Search for a minimal-loss operating spanning tree configuration in urban power distribution systems. In: Proc. of 5th Power Systems Comp. Conf., 1975:1-5.

[6] Peponis GJ, Papadopoulos MP, Hatziargyriou ND. Optimal operation of distribution networks. IEEE Trans. Power Systems, 1996; 11(1):59-67.

[7] Schmidt HP, Ida N, Kagan N, and Guaraldo JC. Fast reconfiguration of distribution systems considering loss minimization. IEEE Trans. Power Systems, 2005; 20(3):1311-1319.

[8] Morton B, Mareels IM. An efficient brute-force solution to the network reconfiguration problem. IEEE Trans. Power Systems., 2000; 15(3):996-1000.

[9] Sitti MW, Nicolae DV, Jimoh AA, Ukil A. Reconfiguration and load balancing in the LV and MV distribution networks for optimal performance. IEEE Trans. On Power Delivery, 2007; 22(4):2534-2540.

[10] Vishwanadha Raju GK, Bijwe PR. An efficient algorithm for minimum loss configuration of distribution system based on sensitivity and heuristics. IEEE Trans. on Power Systems, 2008; 23(3):1280-1287.

[11] Olamaei J, Niknam T, Arefi SB. Distribution feeder reconfiguration for loss minimization based on modified honey bee mating optimization algorithm. Energy Procedia, 2012; 14:304-311.

[12] Arefi A, Olamaei J, et al. Loss reduction experiences in electric power distribution companies of Iran. Presented at: The 2nd International Conference on Advances in Energy Engineering, 2011.

[13] MiPower User Manual, Power Research and Development Consultants Pvt. Ltd, Bangalore, Karnataka, 1998.

[14] Nagendra Rao PS, Ramakrishnan DS, Parthasarathy K. An Algorithm for relocation of distribution transformers. Electric Machines and Power Systems, 1996; 24(7):721-732.

[15] Pabla AS. Electric Power Distribution. 6th ed. TMH Publications, 2011.

[16] Lay outs, Load and Line Details of TC413A11: Operation and Maintenance -2 Manual. Chamundeshwari Electric Supply Company (CESC), Hassan, Karnataka, 2010.

[17] Raghavendra CG, et al. Optimal placement of a distribution transformer for loss minimization. Research report. Malnad College of Engineering, Hassan, 2012. 